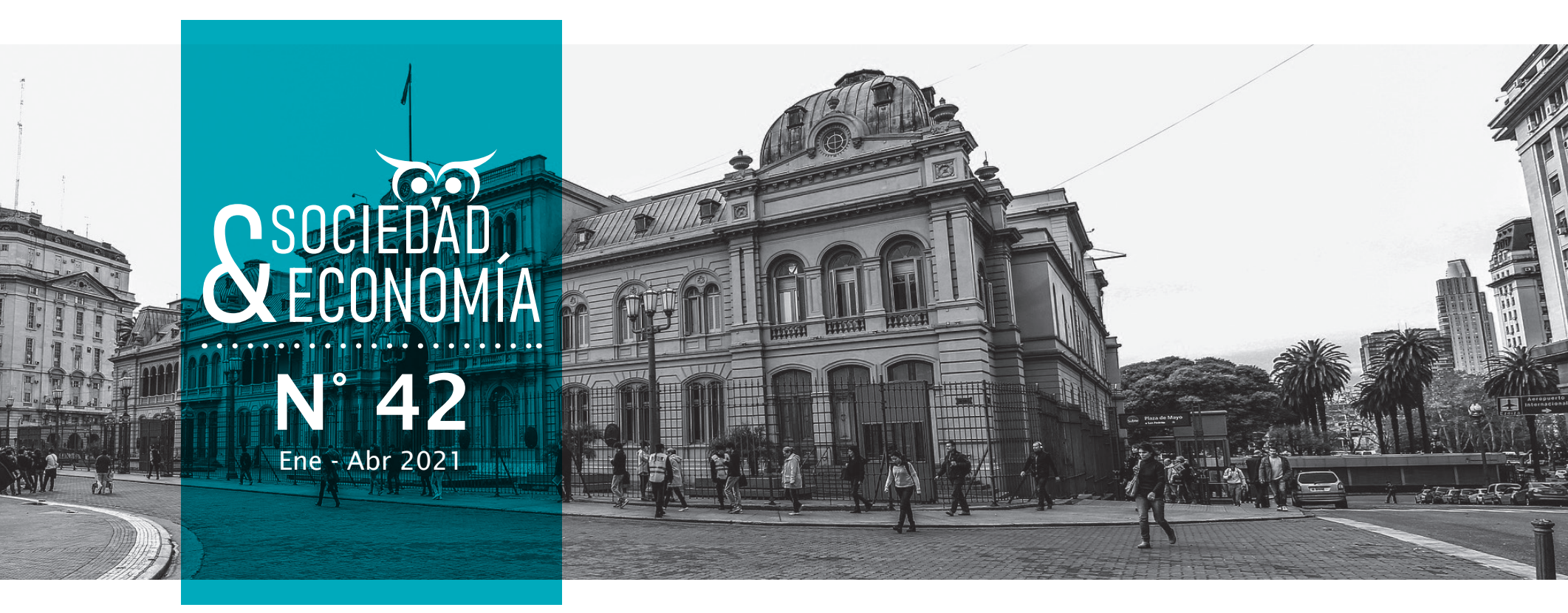

\title{
Estado, estrategias de desarrollo y las ideas “correctas" en América Latina
}

\author{
State, Development Strategies and the "Right" \\ Ideas for Latin America
}

\section{Cristhian Rubén Seiler ${ }^{1}$}

Instituto de Humanidades y Ciencias Sociales del Litoral (Consejo Nacional de Investigaciones Científicas y Técnicas - Universidad Nacional del Litoral), Santa Fe, Argentina.

시 cristhianseiler@gmail.com

(1) http://orcid.org/0000-0002-8893-307X

Recibido: 28-1 1-2019 Aceptado: 07-08-2020 Publicado: 15-02-2021

1 Doctor en Ciencias Sociales. 


\section{Resumen}

En las últimas dos décadas, la persistencia de rasgos periféricos en la estructura productiva de los países latinoamericanos habilitó a cuestionar en qué medida el pensamiento neodesarrollista permitió consolidar un modelo consistente y alternativo al neoliberal. Como estrategia de análisis, el trabajo introduce la dimensión de las ideas y axiomas que orientan la acción estatal en el campo de las políticas de desarrollo. El trabajo es teórico y argumenta que las acciones y estrategias estatales nunca se dan en un vacío, sino en el contexto de ideas y enunciados acerca de cómo alcanzar el desarrollo, los cuales son hegemónicos, específicos y construidos históricamente. Se concluye que las acciones estatales neodesarrollistas no deberían considerarse como fracasos o anomalías institucionales, sino más bien como intervenciones que fueron orientadas por ideas "correctas" que favorecieron la reproducción de los rasgos periféricos.

Palabras clave: políticas de desarrollo; neo desarrollismo; economías periféricas; neoliberalismo.

Clasificación JEL: O19; H11.

\section{Abstract}

Over the past two decades, the persistence of peripheral features in the productive structure of Latin American countries brings into question to what extent neo-developmental thinking has enabled the development of a consistent model as an alternative to the neoliberal model. As an analytical strategy, this work introduces a range of ideas and axioms that guide the State's actions in the field of development policies. This work is theoretical and asserts that state actions and strategies never occur in a vacuum, but rather, are performed in the context of ideas and statements about how to achieve development, which are hegemonic, specific, and historically constructed. It is concluded that neo-developmental actions by the State should not be considered institutional failures or anomalies, but rather, interventions that are guided by "right" ideas that contribute to reproducing peripheral features.

Keywords: development policies; neo-development; peripheral economies; neoliberalism.

JEL Classification: O19; H11.

\section{Financiación}

Este trabajo fue elaborado en el marco de la instancia posdoctoral financiada por el Consejo Nacional de Investigaciones Científicas y Técnicas, Argentina.

\section{Conflicto de interés}

El autor declara no tener ningún conflicto de interés. 


\section{Introducción}

Hace dos décadas, las consecuencias del neoliberalismo en Latinoamérica desencadenaron la "marea rosa" (pink tide) del progresismo latinoamericano, marcada por diferentes reacciones nacionales que fueron denominadas neodesarrollistas (Chodor, 2015). Los resultados en cuanto al crecimiento económico y reducción de la pobreza alcanzados por las experiencias concretas (particularmente, en Argentina y Brasil) daban cuenta de una consolidación neodesarrollista que señalaba el rol preponderante del Estado en la tarea de alcanzar el desarrollo y hacer frente a la hegemonía neoliberal imperante a nivel global (Bresser-Pereira, 2017; Diniz et al., 2012; Katz, 2015). No obstante, en distintos tiempos, las experiencias mostraron restricciones en cuanto a su consistencia, lo que puso límites concretos a su alcance como proyectos o modelos políticos alternativos al neoliberalismo.

La literatura vinculada al capitalismo comparado se propuso capturar analíticamente el desarrollo de estas experiencias y logró captar aspectos relevantes en cuanto a sus rasgos más sobresalientes. Al igual que la matriz fuente de variedades de capitalismo desarrollada en los países centrales por Hall y Soskice (2001), dicha literatura proponía avanzar en el entendimiento de las especificidades institucionales del capitalismo en los países latinoamericanos. De allí, el esquema neodesarrollista surge como un modelo de largo aliento para el desarrollo latinoamericano (Boschi y Gaitán, 2008; Bresser-Pereira, 2017; Diniz et al., 2012; Gaitán y Boschi, 2016). Sin embargo, cuando se observa la persistencia de rasgos como la transnacionalización de la economía o la dependencia de exportaciones basadas en recursos naturales (rasgos representativos de la era neoliberal de fines del siglo pasado), es posible hacer cuestionamientos al potencial de dichos modelos, tanto en su dimensión analítica como política (Féliz, 2015; Katz, 2015).

En ese marco, el trabajo busca explorar algunos elementos que no han sido incorporados en el marco analítico mencionado. En otras oportunidades, algunos trabajos han señalado la importancia de trascender la explicación mono-escalar que subestima el marco global del sistema capitalista y las formas específicas que adquieren las políticas de desarrollo en los países periféricos (Ebenau, 2012; Fernández et al., 2018). Pero resulta significativo reafirmar dicha perspectiva y colocar a las trayectorias nacionales recientes en el contexto más amplio del imaginario neoliberal e introducir la dimensión de aquellas ideas que influyen decisivamente sobre los diseños de políticas de desarrollo y, al mismo tiempo, buscan ampliar la hegemonía del capital global y su influencia sobre las naciones.

Si bien la agenda neodesarrollista remedió algunas falencias de la literatura clásica de variedades de capitalismo, en menor medida analizó el modo en que los actores globales hegemónicos condicionan las trayectorias de desarrollo mediante las ideas que alimentan buena parte de las políticas de desarrollo de las experiencias analizadas. Concretamente, se hace referencia al modo en que organismos internacionales de crédito y apoyo al desarroIlo (OIC) (por ejemplo, BM, BID, entre otros) han difundido ideas particulares que han penetrado e incidido en la conformación de particulares políticas públicas de desarrollo. Esto puede visualizarse en la conformación de un sentido común global o ideas "correctas" acerca de cómo alcanzar el desarrollo en contextos periféricos. Tal es el caso de una "competitividad correcta" afín a la "obsesión exportadora" persistente en América Latina.

La introducción de estos elementos permite advertir que cuando se analiza la intervención estatal y sus resultados sin el debido contexto de referencia y los fundamentos ideacionales de la acción, es decir, sin detallar pormenorizadamente el sentido e ideas hegemónicas y los supuestos detrás de la racionalidad de la acción, la reflexión puede caer en conclusiones del tipo "más o menos" intervención. Pero, cuando se da atención a los detalles -y las ideas que orientan la acción de los Estados vienen a un primer plano- es posible superar esa disyuntiva y concluir que lo que a primera vista se presenta como una falla de la intervención estatal, en realidad puede argumentarse que es la expresión visible de enunciados 
y supuestos que orientan la acción. Por esta razón, la fuerza de la hegemonía global y las ideas que circulan no son elementos neutrales al momento de evaluar el papel estatal en las trayectorias de desarrollo en contextos periféricos.

El trabajo se organiza en cinco partes. Primero, se hace un repaso de la literatura en la que se inscriben los análisis neodesarrollistas, y se señalan brevemente las limitantes que presentan estos estudios. Segundo, presenta cómo en diferentes momentos históricos el marco hegemónico que excede el ámbito nacional ha influenciado las acciones del Estado y las trayectorias nacionales a través de ideas particulares; tanto la influencia de las ideas de mediados de siglo XX ancladas en la articulación virtuosa entre Estado y desarrollo, como la hegemonía del mercado de fines del siglo. La tercera parte señala a las ideas como objeto relevante de la configuración de la acción estatal, dado el sentido común que se establece como marco de referencia acerca de cómo alcanzar el desarrollo. En la cuarta parte, se señalan los mecanismos y actores que han vehiculizado ideas particulares de desarrollo centradas en una "competitividad correcta". Y, en la quinta parte, se presentan algunas evidencias de la afinidad entre las ideas utilizadas y la reproducción de ciertas características de la dinámica y la trayectoria periférica. Por esa razón, la intervención estatal no debería considerarse una falla, sino un modo particular de operar que es coherente con una visión persistente de globalización. Finalmente, las conclusiones.

\section{Revisando el capitalismo del centro a la periferia}

A finales del siglo XX, la tesis de la convergencia hacia un modelo único y universal de capitalismo fue cuestionada por la literatura de las variedades de capitalismos. Dicha literatura señala que existen diversas formas de organización institucional del sistema capitalista, y que estas son el resultado de diferentes trayectorias históricas y comportamiento de los agentes sociales. El trabajo de Albert (1993) estimuló tempranamente un esquema de in- terpretación de dos modelos diferenciados: el neo-americano y el renano. Esta dualidad indicaba una novedad: la coexistencia de diferentes economías de mercado. Bajo la misma premisa de coexistencia de modelos, la distinción planteada por Albert fue refinada en torno al clásico de Hall y Soskice (2001), que alcanzó mayor comprensión de la diversidad capitalista a través de las nociones de economía de libre mercado (ELM) y economía de mercado coordinado (EMC).

Focalizando sobre la estructura institucional, sus relaciones, los actores y las políticas que daban coherencia al sistema, para Hall y Soskice los modelos se comprenden tipológicamente de acuerdo a la forma en que se generaban las complementariedades institucionales entre cinco esferas de acción: a) relaciones industriales; b) sistema educativo y formación profesional; c) gobierno corporativo; d) sistema financiero; y e) relaciones entre empresas. Si la resolución de la interacción de las esferas mencionadas se realiza a través de una complementariedad positiva, mediada por el mercado, o a través de un proceso más coordinado de fuertes instituciones (Estado, sindicatos, etc.), la formación capitalista específica tenderá a asociarse a uno u otro de los dos tipos-ideales presentados anteriormente: ELM o EMC; o, en todo caso, será una típica economía de mercado mixta (EMM), que hará transición hacia uno u otro modelo de acuerdo a la modalidad en que se resuelva la complementariedad.

En el campo del capitalismo comparado de los países desarrollados, el avance de esta literatura presentó tres importantes aportes: a) las economías nacionales constituyen configuraciones sistémicas que generan determinados comportamientos de los actores; b) los arreglos institucionales pueden constituir ventajas positivas o negativas para dichos comportamientos; y c) las diversas formas capitalistas responden a trayectorias históricas (Aguirre y Lo-Vuolo, 2013; Fernández y Ebenau, 2018).

Con la aspiración de ampliar el potencial explicativo, el análisis de las variedades capitalistas complejizó su enfoque y otras latitudes fueron 
objeto de análisis (Ebenau et al., 2015). Por ejemplo, Schneider (2009a; 2009b) y Schneider y Karcher (2012) ampliaron la tipología dual de la literatura de variedades de capitalismo al abordar los países latinoamericanos (principalmente Argentina, Brasil y Chile), destacando la preeminencia de una economía jerárquica de mercado (EJM). Estos autores han mostrado cómo el peso de las empresas transnacionales (ETN) y los grandes actores económicos nacionales ha inducido a una configuración capitalista, basada en actividades trabajo-intensivas, con mercados de trabajo flexibles, fragmentados y de baja formación, con alta informalidad, producto de la escasa densidad productiva. De esta manera, a diferencia de la complementariedad positiva reconocida en los modelos ELM y EMC, en el contexto latinoamericano se daba una complementariedad negativa, que explicaría en buena medida la heterogeneidad estructural y el paupérrimo performance en innovación y desarrollo (Schneider y Karcher, 2012).

A pesar de la ampliación tipológica y geográfica, esta innovación conceptual no dejó de reproducir las insuficiencias teóricas que le habían sido criticadas desde el centro: el enfoque funcionalista centrado en las empresas; el profundo nacionalismo metodológico, y su enfoque a-histórico (Ebenau, 2012); la centralidad del equilibrio por sobre el cambio en las trayectorias; y la falta de especificidad que posee el Estado para dar cuenta de la configuración institucional, y la propensión al cambio de la trayectoria (Fernández et al., 2018).

Respondiendo -al menos parcialmente- a estas críticas, un grupo de investigadores dirigido por Renato Boschi (Boschi, 2011; Boschi y Gaitán, 2008; Diniz et al., 2012) instó a dar continuidad a estos estudios, apoyando sus explicaciones en el institucionalismo estatista surgido de interpretaciones heterodoxas de las experiencias del sudeste asiático (EA) y el pensamiento neodesarrollista emergido recientemente en Latinoamérica.

Bajo sus reflexiones, el Estado sería capaz de resolver eficazmente los problemas del accionar colectivo prevaleciente a través de la implementación de una visión de largo plazo bajo un "proyecto nacional de desarrollo inclusivo", que daría consistencia no solo a la competitividad empresarial y desempeño macroeconómico, sino también al mejoramiento de las condiciones socioeconómicas de la población (Bresser-Pereira, 2017). Bajo la mirada del "nuevo desarrollismo", la clásica discusión acerca de dónde colocar el motor del crecimiento de los países, inclinó la balanza hacia el modelo orientado a las exportaciones (export-led) (Bresser-Pereira, 2012), que hizo compatible la obsesión exportadora abierta por el neoliberalismo y un papel activo del Estado.

Si bien esta emergente literatura resultó útil para incorporar elementos significativos (centralidad de la intervención estatal o bien la importancia de la trayectoria nacional en los resultados de desarrollo), ha desconsiderado la dimensión global del sistema capitalista y las especificidades que asumen los espacios periféricos (Fernández et al., 2018). Dentro de los cuestionamientos se puede mencionar que, por un lado, consideran que las trayectorias de los países surge como auto-construcción, lo cual relega una interpretación más precisa y holística de las condiciones geopolíticas del funcionamiento del capitalismo, en donde tienen lugar las variaciones capitalistas (Crespo y Muñiz, 2017; Fernández et al., 2017); y, por otro lado, desestima la estructura, potencia y la persistencia de la hegemonía neoliberal en la construcción de un sentido común a nivel global acerca de cómo alcanzar el desarrollo en los espacios periféricos (Cypher, 2007; Fernández, 2015).

Sobre la base de estas críticas, a continuación, se introducen algunos aspectos que permiten un análisis más completo de las variedades capitalistas en la periferia, los cuales pueden aportar elementos de juicio y aproximar explicaciones novedosas acerca del porqué la centralidad estatal de los proyectos neodesarrollistas no ha conducido a una variación de la variedad capitalista en los países latinoamericanos (Fernández, 2017). En este sentido, la centralidad del Estado y su papel en la transformación productiva de la región (tanto para el crecimiento económico como para la distri- 
bución del ingreso y la inserción internacional de los países) podría ser mejor entendida en un marco más amplio que evalúe las ideas que circulan y fundamentan las intervenciones y las políticas públicas de desarrollo. Sobre esto tratarán los siguientes apartados.

\section{Transformaciones del capitalismo, ideas y políticas en su contexto}

Las reflexiones del desempeño de los países y la conformación de variedades capitalistas en la periferia deben ser comprendidas en el marco de un análisis más amplio, que entienda al capitalismo bajo una lógica sistémica que configura al globo en centros y periferias (Arrighi, 1978; Prebisch, 1986). Un breve recorrido histórico permite advertir la utilidad de introducir el contexto y las ideas que estimularon ciertas configuraciones capitalistas.

Durante las estrategias desarrollistas de mediados del siglo XX, los países desafiaron el esquema centro-periferia existente a través de diferentes estrategias que colocaron al Estado en el centro de la escena del proceso de transformación productiva, con epicentro en proyectos nacionales (Cypher, 2007). Los avances ya conocidos del Este Asiático y la profunda modernización de los países de América Latina exhiben un proceso de formación de instituciones domésticas que otorgaron centralidad al Estado para estimular el crecimiento y direccionarlo en función de la transformación industrial de sus economías, dado el paradigma de desarrollo de ese momento (Chang, 2010; Chibber, 2002; Gore, 1996).

Si bien la conceptualización de los estados desarrollistas da cuenta del papel estratégico del Estado y sus atributos institucionales, exacerba el carácter nacional de su construcción y pretendido carácter universalista para todo tiempo y lugar. Por una parte, la centralidad estatal, a través de los procesos de planificación y desarrollo industrial, también fue la expresión interna de un paradigma dominante y de procesos más amplios de formación estatal propios de las condiciones geopolíticas del capitalismo en que tuvieron lugar, entre ellas la benevolencia norteamericana y su expresión en diferentes contextos geográficos (Gore, 1996; Yeung, 2017). Es decir, es posible que diferentes países expuestos a un mismo contexto histórico no reaccionen del mismo modo, dada la correlación de fuerzas internas que determina la dirección del proceso.

Por ejemplo, en las experiencias asiáticas (particularmente, Corea del Sur), Chibber (2003) señala que la estrategia de desarrollo se vio favorecida por una suerte de consenso idea-fuerza de la necesidad de una transformación industrial dirigida por el Estado que habilitó la construcción de capacidades estatales. En cambio, en América Latina, los procesos de posguerra fueron más abiertos a alternativas $u$ opciones de desarrollo que introdujeron contingentes de especialistas y expertos al Estado, pero que no lograron una profesionalización plena más allá de variaciones notables de sus capacidades (Sikkink y Wolfson, 1993). Este proceso tuvo un particular impulso desde la CEPAL en común acuerdo con gobiernos nacionales y otros OI (en particular el BID), que movilizaron la necesidad de profesionalización estatal e incorporación de la planificación económica en la región (de-Mattos, 1979).

Tomando las tres décadas posteriores a la segunda posguerra, es posible acordar que, más allá del grado de calidad alcanzado por el Estado, lo cierto es que el carácter desarrollista y las estrategias de transformación industrial se dieron bajo ciertas condiciones irrepetibles históricamente que le fueron propicias (Fernández et al., 2017). El ideario de desarrollo colocaba a los procesos de industrialización encabezados por el Estado como el axioma de transformación de los países menos desarrollados (Cypher, 2007).

La apreciación del contexto y las ideas hegemónicas que puede parecer obvia, adquiere más fuerza ante el cambio de contexto geopolítico e ideas emergente del paradigma neoliberal que transformó los aparatos estatales y los desplazó de los procesos de desarrollo (Crotty y Lee, 2005; Hall, 1993; Jessop, 2016). El triunfo de una geopolítica de disciplina 
neoliberal (Gill, 1995) brindó ideas alineadas con la supremacía del mercado como mejor asignador de los recursos. El Estado ya no es presentado como actor fundamental del desarrollo (cargando el estigma de no adecuarse a las nuevas condiciones de funcionamiento del capitalismo), pero sí tiene un papel clave en la libre circulación de los flujos globales y el sostenimiento del modelo global (Crotty y Lee, 2005; Cypher, 2007; Weiss, 1998).

Ese fundamento ideológico sedimentó la consolidación de un capitalismo global que no estimula la formación de procesos nacionales de desarrollo traccionados por Estados, sino, por el contrario, abona la "obsesión exportadora" de los países periféricos como la clave orientadora del desarrollo (Cypher, 2007; Neilson, 2014). Esa obsesión se articuló con la novedosa organización de la producción que ha tendido a articularse cada vez más en cadenas globales de valor (CGV) gobernadas por el capital transnacional (Gereffi, 2014). Desde entonces, se aduce que el avance tecno-productivo y social de las empresas y países debe buscarse en dichas cadenas y en la circulación de los flujos productivos a escala global como la fuente privilegiada de oportunidades. De modo que aquel proceso emergente, iniciado a fines del siglo $X X$, en las primeras décadas del siglo XX y bajo un proceso de teorizaciones, pasó a constituirse en el fundamento ideológico y directivo de políticas e instrumentos de las intervenciones estatales (Fernández, 2015; Fernández y Trevignani, 2015; Neilson, 2014).

En este contexto, desafiar la posición de los países periféricos en las cadenas implica señalar dos aspectos limitantes. Por un lado, dentro del paradigma de desarrollo imperante, el Estado ocupa un rol poco relevante para alterar el comportamiento económico, ya que este pasó a depender cada vez más de las dinámicas productivas que se presentan como dadas; $y$, por otro lado, debido a la organización tecnoproductiva propia de dichas cadenas, ya que las funciones y actividades de mayor retención de valor son controladas selectivamente por las empresas y espacios centrales en los que se encuentran las casas matrices (Kaplinsky, 2000), independientemente de la capacidad de organización local (Milberg, 2008). De modo que lo que se ha presentado como una oportunidad para el desarrollo de los países, en realidad contiene un profundo carácter contradictorio de jerarquización y subordinación de empresas y sectores que reproduce la estructura centro-periferia (Fernández y Trevignani, 2015; Neilson, 2014).

Tanto la reconfiguración de la dinámica capitalista de las últimas tres décadas como las narrativas e ideas emergentes que le han dado sustento político e ideológico a la primacía del mercado y la desarticulación estatal, son parte de un proceso de cambio cultural y reconfiguración del poder de las naciones en favor del capital transnacional (particularmente, financiero) y la disminución de las posibilidades de alterar las trayectorias de desarrollo en los espacios periféricos (Fernández, 2017; Sum y Jessop, 2013).

\section{Ideas y políticas de desarrollo en el contexto de la globalización}

La persistencia del nacionalismo metodológico en el análisis neodesarrollista coloca al Estado como un actor central del cambio de las trayectorias de desarrollo, sin realizar un debido reparo en las dimensiones señaladas anteriormente. Al igual que las reflexiones realizadas por Schneider, la visión neodesarrollista prescinde en sus análisis de la importancia de un marco más general de funcionamiento sistémico del capitalismo y el modo en que el paradigma hegemónico de desarrollo condiciona los movimientos posibles de las trayectorias nacionales.

En el fondo, a la luz de las preocupaciones de la perspectiva neodesarrollista, los efectos de las complementariedades negativas señaladas por Schneider (primacía del capital transnacional y el carácter de trabajo intensivo de las economías), no necesariamente serían defectos o contradicciones del modelo neodesarrollista, sino más bien el resultado de asumir más o menos implícitamente una determinada visión de cómo alcanzar el desarrollo en los países 
periféricos en un contexto neoliberal que provee ideas, herramientas y políticas poco familiares con el desarrollo nacional.

Por esta razón, es posible acordar que el ascenso de los gobiernos más estatistas en América Latina fue condición necesaria, pero no suficiente (Monedero, 2019), ya que no todo lo que se emprendió bajo un "proyecto de desarrollo nacional" fue expresión de ideas y herramientas políticas que hicieran prosperar la dimensión económica nacional y la complejidad de la estructura productiva. Por ejemplo, la experiencia argentina de las últimas dos décadas expone que la proyección neodesarrollista de reindustrialización no logró revertir el dominio de los actores transnacionales, ni su matriz exportadora de productos primarios (Bekerman et al., 2018; Belloni y Wainer, 2013).

De este modo, el análisis de las ideas y fundamentos conceptuales de las políticas de desarrollo y la intervención estatal permitiría entender mejor el papel, el contenido, la orientación y la potencia del abordaje estatal en un asunto clave como es el aspecto productivo frente a la persistencia de los problemas estructurales en América Latina (mencionados anteriormente). Indagar este registro ideacional no solo permite identificar las relaciones causales de los problemas que se establecen como más verosímiles, sino también la determinación del cómo debe ser alcanzado el desarrollo (Blyth, 2002; Sum y Jessop, 2013).

Aunque un análisis de este tipo a menudo se asocia más a teorías, conceptos y dimensiones analíticas que poco tienen que ver con fenómenos concretos, en realidad la acción del hombre, así como la de los Estados, no es indiferente al conjunto de enunciaciones teóricas o ideacionales que otorgan sentido a la acción y, en este caso particular, condicionan y/o determinan los medios, la fisonomía y el conjunto de herramientas e instrumentos utilizados por los gobiernos para intervenir en los asuntos públicos.

Es por esta razón que al revisar las ideas que orientan y dan sentido a las intervenciones neodesarrollistas, este trabajo procuró no con- siderar que tales experiencias contenían aspectos institucionales fallidos. Aunque esto no pretende decir que América Latina posee aspectos institucionales que requieran ser fortalecidos. El punto a señalar es que las experiencias buscaron concretar proyectos con ideas hegemónicas que fueron consideradas "correctas" para alcanzar el desarrollo. Y, por tanto, lo que se podría considerar como fallido, en realidad es parte del cumplimiento efectivo de objetivos implícitos o explícitos más amplios, que obedecen a intereses de otros actores y dan consistencia a la configuración capitalista contemporánea, en la cual los países latinoamericanos permanecen y reproducen una posición periférica.

Ahora bien, frente a lo señalado, a continuación, se expone evidencia acerca de la incidencia de dichos aspectos en la configuración de las intervenciones públicas en la periferia, resaltando las ideas que han primado y a través de qué actores esto ha sido posible.

\section{La banca multilateral y las ideas "correctas" para el desarrollo}

Desde mediados del siglo $\mathrm{XX}$, los organismos internacionales de crédito (OIC) (por ejemplo, BM y BID) tuvieron un papel importante como instancias de germinación y difusión de ideas de desarrollo, pero también de fortalecimiento de los Estados latinoamericanos. No obstante, dentro de la profunda transformación neoliberal y acorde al contexto, los OIC incidieron en la configuración de las políticas de desarrollo de cada país y en deshabilitar la centralidad de los Estados nacionales. No es una relación de dominación pura y simple por parte de dichos organismos, sino que existió un marco de saneamiento de las cuentas nacionales en el que penetraron discursos, ideas, marcos conceptuales e incluso formatos institucionales acordes al contexto global de hegemonía neoliberal.

En este sentido, las intervenciones estatales fueron flanqueadas, penetradas (algunas más que otras) o simplemente configuradas por ese orden hegemónico que estableció un 
"sentido común" acerca de las formas en que ciertos asuntos ingresan a la agenda y se llevan adelante para su resolución (Cox, 2013; Gill, 2012; Sum y Jessop, 2013). Si bien este proceso no estuvo exento de conflictos y resistencias locales, el orden neoliberal actuó disciplinarmente sobre las economías periféricas y sus instituciones (Gill, 1995; Gill y Law, 1989; Wade, 2006), para dar de baja la centralidad estatal sobre la que se edificaron las economías nacionales de posguerra (Dicken et al., 2001; Fernández, 2001).

El desplazamiento del Estado y la preeminencia del mercado como mejor asignador de recursos, marcó el curso del aprendizaje de los organismos estatales que pasaron a operar como puentes entre la circulación global de ideas y la implementación de instrumentos pro mercado (Peck, 2010). Desde entonces, el uso de dichas ideas forjó un círculo de afinidad entre consultores, funcionarios y las estructuras burocráticas, que desarrolló una modalidad de intervención y se apoyó en la idea "correcta" de competitividad para el desarrollo (Neilson, 2014; Sum y Jessop, 2013).

Argentina puede ser un buen ejemplo de esto. Es relevante observar la incidencia del BID o del BM sobre instrumentos de política pública o en la configuración de instituciones vinculadas al desarrollo en este país. Desde inicios de la década de 1990, donde la idea de desarrollo tuvo un cambio radical respecto a las décadas previas (Fernández et al., 2006), las estrategias de modernización económica y fortalecimiento de las pequeñas y medianas empresas, o bien la reconfiguración de las acciones e instituciones del campo científico-tecnológico, han sido iniciada o soportadas por OIC, principalmente por el BID y su estrategia de desarrollo empresarial (Aguiar et al., 2015; Seiler y Fernández, 2017). Sin cuestionar la apertura financiera y comercial que profundizó los procesos de extranjerización y reprimarización de la economía argentina, los OIC pujaron diferentes transformaciones sobre la idea de que el desarrollo sería posible a través de las oportunidades que brindaban la globalización y los actores globales (Baruj y Porta, 2006; BID, 2005). El abanico de políticas públicas que surgió no se caracterizó por un enfoque de cambio de las estructuras productivas con centralidad estatal (Dini et al., 2014).

Durante las experiencias neodesarrollistas, estas operaciones fueron similares. Las políticas orientadas a pymes o al campo científico-tecnológico han tenido una clara afinidad con las ideas difundidas por los OIC de relevancia en la región, mientras los organismos públicos vinculados al desarrollo no asumieron una marcada centralidad (Aristimuño y Lugones, 2019; Lavarello, 2017; Seiler, 2020; Seiler y Fernández, 2017). En la última década y media, los OIC han fortalecido la idea de que, en el ámbito de la producción organizada en cadenas globales de valor (CGV), el desarrollo viene de fortalecer a las pymes y/o aglomeraciones de empresas e incentivarlas a participar en esas cadenas (Fernández, 2015). En la nueva configuración de la producción global, las pymes obtendrían acceso a nuevos mercados, así como a amplios beneficios de las empresas líderes -transferencia tecnológica, innovación, etc.- que permitirían la actualización tecnológica necesaria para el crecimiento y desarrollo de la economía en general (Hernández et al., 2014; Pietrobelli y Rabellotti, 2011; Stezano, 2013; UNCTAD, 2010).

Esta plataforma de ideas que, en buena medida, ha surgido como fuente de las políticas de desarrollo de las últimas tres décadas, no contempla aspectos referentes a las contradicciones propias de las relaciones capitalistas o, por ejemplo, aquellas que se dan al interior de las CGV (Werner et al., 2014). De este modo, cuando los Estados asumen ideas y marcos conceptuales asociados a este conjunto de enunciados para orientar su acción, están asumiendo todos los supuestos, objetivos implícitos e intereses que son permeados por teorizaciones y operaciones de abstracción necesarias para constituirlas en herramientas prácticas de dominación y subordinación (Fernández, 2015). Por esta razón, cuando se evalúa el papel del Estado y no se tienen en cuenta estos aspectos sensibles al contexto, los análisis más agregados de la intervención estatal pueden caer en conclusiones erradas o divergentes. 
En el apartado siguiente, se presenta más detalladamente la potencia de las ideas y abstracciones que han sustentado las políticas de desarrollo en las últimas dos décadas.

\section{6. "Competitividad correcta" a través de cluster y la fast industrialization}

Desde mediados de 1990, el consenso entre la academia y los hacedores de políticas de desarrollo dio centralidad a ideas y esquemas conceptuales, señalando que la competitividad de la economía venía por introducir a las pymes en la dinámica de los mercados globales, tanto individualmente como a través de la constitución de clusters de empresas (Fernández et al., 2008). El BM y el BID (así como otras instituciones regionales como la (EPAL) se tornaron multiplicadores y nodos de articulación de ese imaginario.

El herramental teórico de las economías de aglomeración centradas en el esquema conceptual de clusters, no solo fue un marco analítico hegemónico en los ámbitos académicos del desarrollo regional, sino que rápidamente fue acogido por los OIC como base de las políticas de desarrollo que pasaron a fundamentar las estrategias de los países en la periferia (Fernández et al., 2008). Dicho esquema resalta las regiones/localidades, reconociendo los agrupamientos armónicos y dinámicos de pyme que aseguran cohesión y competitividad por medio de las relaciones de cooperación-interacción. A diferencia de las estrategias top-down, de las formas centralizadas y dirigidas por el Estado, este paradigma resalta la construcción de economías de aglomeración sustentadas en la lógica bottom-up, que suponen que arreglos institucionales descentralizados territorialmente pueden de manera autónoma viabilizar el desarrollo (Fernández y Vigil, 2007; Werner et al., 2014).

Cuando los organismos estatales asumen este esquema, configuran una modalidad de intervención en la que no solo la escala nacional resulta poco relevante y estratégica para fortalecer la dimensión pyme y sus encadenamientos territoriales, sino que el Estado nacional es desplazado a un papel facilitador -y ensamblador de programas- para desencadenar la cooperación entre actores (Fernández y Vigil, 2007). Paradójicamente, despojada su centralidad, asumiendo que la globalización traerá frutos, el Estado actúa (re)valorizando los espacios productivos regionales/locales solo para operar globalmente $\mathrm{y} / \mathrm{o}$ viabilizar su competitividad localmente situada, como proveedores del capital global (Werner et al., 2014).

En este escenario, pasa por inadvertida la importancia que posee la escala nacional (estatal y de acumulación) en la formación de encadenamientos aguas arriba y abajo, y con los procesos de aprendizaje internos (Fernández, 2010; Furtado, 1974; Hirschman, 1968). Resulta desestimada al punto que el imaginario clusters orienta la acción estatal a reproducir un marco de múltiples enclaves productivos e islas de competitividad fragmentadas territorialmente e incapaces de articularse a nivel nacional (Fernández, 2010; SELA, 2012), pero insistentemente invitadas a vincularse a los flujos globales controlados por el capital transnacional (Bekerman et al., 2013).

Paralelamente, el concepto de cadenas globales de valor (CGV) fue ganando importancia como complemento internacional al cuerpo teórico de clusters (Neilson, 2014; Werner et al., 2014). A inicios de los 2000, al tiempo que el enfoque clusters ganaba aceptación, en los espacios académicos internacionales fue asociado con las proposiciones del enfoque de CGV (Werner et al., 2014). Esta dupla dio consistencia práctica a los axiomas sustentados en la glocalization (Swyngedouw, 1997). De este modo, la inserción de las empresas en las CGV se presentó como un paso subsiguiente y necesario en la estrategia de desarrollo para la actualización industrial y tecnológica (upgrading), ya que esto incorporaba a las empresas y economías de aglomeración en potenciales curvas dinámicas de aprendizaje e innovación (Neilson, 2014).

El axioma práctico de las CGV, difundido por los OIC, sostiene que la estrategia más favorable para los países periféricos que no 
controlan las cadenas productivas consiste en promocionar la "inserción rápida" de sus empresas en las CGV ya constituidas, para derivar de ello las "mejoras" en sus estructuras industriales (Baldwin, 2011; Blyde, 2014; Hernández et al., 2014; UNCTAD, 2010). Los beneficios de esta estrategia serían mayores a los que se obtendrían mediante la creación y construcción de encadenamientos (aguas arriba, aguas abajo, al decir de Hirschman), originados por una política industrial explícita o tradicional que prioriza el nivel doméstico nacional (Baldwin, 2011; 2013). La inserción rápida de empresas que muestran competitividad internacional atraería los beneficios de una rápida industrialización (fast industrialization) (Baldwin, 2013; Stezano, 2013). Así, bajo una suerte de complementariedad, los propulsores del enfoque clusters, argumentan que la falta de desarrollo y progreso competitivo de las empresas debe revertirse aprovechando las oportunidades y presiones que ofrece la participación en las CGV, y la colaboración de los proveedores y compradores extranjeros (Giuliani et al., 2005; Hernández et al., 2014; Pietrobelli y Rabellotti, 2011).

Al igual que el enfoque clusters, la competitividad en el marco de CGV es un aspecto ubicado a nivel de empresa, que ignora cuestiones estructurales más amplias, acerca de cómo se produce y reproduce la desigual creación y distribución del valor en las cadenas productivas (Bair, 2005; 2019). Básicamente, el carácter neoliberal del esquema conceptual de CGV hace posible que el capital transnacional tenga acceso a determinados proveedores de insumos estratégicos esparcidos globalmente donde, y solo de ese modo, la fragmentada y desarticulada trama de pequeñas y medianas empresas adquiere importancia estratégica (Ferrando, 2013). Es decir, como orientación de la acción estatal, la clusterización beneficia a las empresas que presentan condiciones y factores productivos estratégicos (bajos costos de la materia prima y mano de obra, infraestructura, etc.), en la medida en que clausura o cierra los procesos productivos de bienes y solo a escala global (Bair, 2005; Fernández, 2015; Neilson, 2014).
Bajo este funcionamiento de valoración selectiva de los factores productivos, los beneficios "potenciales" que ganarían las empresas al introducirse en las cadenas no contemplan el nuevo esquema de dominación, por el cual las condiciones estructurales centro-periferia son mantenidas o reproducidas. Por un lado, debido al control que ejercen las casas matrices de aquellas actividades centrales retenidas en los países de origen (diseño, patente, organización de las redes, precios de comercialización, posventa, entre otros) y, por otro lado, debido a la desarticulación de entramados productivos que se producen en los países periféricos, de aquellos que quedan por fuera de los factores productivos valorados por las empresas transnacionales (Fernández y Brondino, 2018; Fernández y Trevignani, 2015; Kaplinsky, 2000).

De este modo, el conjunto de instrumentos derivado de estas ideas y axiomas ha generado una particular intervención estatal que ha sido afín a la "obsesión exportadora" latinoamericana. Básicamente, consiste en acondicionar un abanico de opciones productivas (fragmentadas, sin densidad nacional, etc.) para ser vinculadas a las empresas líderes que controlan las CGV. Bajo estas directrices, la intervención estatal difícilmente pueda construir eslabonamientos horizontales y verticales a nivel nacional, que es donde es necesario acumular aprendizajes productivos y tecnológicos para alterar la trayectoria de desarrollo (Cimoli et al., 2017).

\section{Consideraciones finales}

La literatura de variedades de capitalismo realizó avances significativos en materia institucional, dentro de los cuales se encuentra haber presentado al modelo neodesarrollista como una alternativa al neoliberalismo. En buena parte, siguiendo la tradición comparativa para señalar que la hipótesis de la convergencia hacia un modelo capitalista único no resultaba evidente, los teóricos neodesarrollistas resaltaron la centralidad del patrón de intervención estatal en las experiencias latinoamericanas como un modelo alternativo a la configuración neoliberal de las décadas pasadas. No obstante, más allá de algunos avances económicos 
y sociales significativos durante las experiencias, ciertos atributos estructurales de la dinámica periférica (extranjerización y reprimarización, principalmente) fueron profundizados siguiendo el retrato que Ben Schneider había señalado en su modelo de economía jerárquica de mercado, gestado en las últimas décadas del siglo XX. De esta forma, si bien el modelo estatista-neodesarrollista presentó importantes avances en la literatura, mantuvo algunas debilidades explicativas que no permitieron dar cuenta de tal configuración, siendo que el liderazgo estatista debía plasmar una trayectoria divergente a la neoliberal.

Básicamente, en el recorrido del trabajo, el objetivo fue introducir la dimensión de las ideas y aquellos axiomas que orientan o inciden en la dirección de las acciones estatales en el campo del desarrollo. Como una estrategia analítica para superar el nacionalismo metodológico, el campo de las ideas, y su dimensión contextual de producción y referencia, dio lugar a sostener que las acciones deben ser analizadas más en detalle desde el punto de vista de las ideas que las fundamentan y orientan, y su vínculo con la dinámica conflictual del capitalismo contemporáneo.

En esta línea, la hipótesis que estimuló el recorrido del trabajo fue considerar que la persistencia de una trayectoria de desarrollo con rasgos periféricos no necesariamente -o solamente- obedecía a ciertas anomalías, fallas o falta de capacidades que podían rastrearse en la dimensión institucional y de forma directa en los recursos estatales. En realidad, al introducir la dimensión ideacional, era posible reconsiderar el papel estatal desde una nueva perspectiva que indicaba que las ideas y axiomas, detrás de las políticas, incentivaron la reproducción de los rasgos periféricos de la estructura productiva que se esperaba que el neodesarrollismo transformara.

Colocar a las ideas en un primer plano no solo implicó traer ciertos enunciados, narrativas, conceptos o axiomas que sustentan una determinada racionalidad de la acción en el contexto neoliberal, sino que también involucró llamar la atención acerca de los mecanismos y actores que entran en juego operando sobre la difusión y aceptación de dicha racionalidad. En este sentido, la evidencia muestra que no es posible entender la dinámica y trayectoria periférica latinoamericana sin hacer referencia al protagonismo de la banca multilateral que se ha constituido en una poderosa usina de pensamiento y difusora de ideas, en diferentes direcciones de acuerdo al momento histórico, pero siempre con una fuerte incidencia sobre la orientación de las acciones estatales. Como se mencionó, la hegemonía neoliberal señala los caminos más apropiados para alcanzar el desarrollo y es más efectiva en aquellos espacios nacionales donde las estructuras estatales carecen de atributos y el carácter institucional suficiente para resistirlas.

Para mostrar estos aspectos, la noción de una "competitividad correcta" que encierra una orientación de racionalidad de la acción estatal en el plano productivo, permitió ordenar conceptos, supuestos, enunciados e incluso actores y mecanismos de difusión y aceptación de los mismos en el contexto de la globalización. Mientras las ideas o axiomas asociados al desarrollo nacional liderado por el Estado fueron cuestionados y desacreditados, la articulación de conceptos como el de clusters y la inserción en los flujos de producción global se alinearon con la "obsesión exportadora" asumida por el modelo neoliberal $y$, posteriormente, por el neodesarrollista, para debilitar la potencia de las acciones en materia de transformación estructural. Dadas las debilidades y el derrotero estatal para formular una estrategia endógena y más autónoma, esta combinación fundamentó políticas de desarrollo que han sido el complemento local para ensamblar selectivamente segmentos productivos locales en el circuito del capital transnacional y la dinámica de acumulación a escala planetaria.

Esto muestra que, aunque el modelo neodesarrollista colocó al Estado como epicentro decisional, las opciones estratégicas que dieron sentido a la acción no solo no tuvieron la suficiente potencia, sino que tampoco fueron neutrales. Por lo tanto, cuando la acción estatal se observa desde un punto de vista más minucioso, percibiendo las ideas y axiomas que le dan 
sentido a los diferentes movimientos $y$, al mismo tiempo, se contextualizan en un campo hegemónico más amplio, es posible detectar en qué grado y a qué intereses contribuye su accionar.

Entonces, al incorporar analíticamente las ideas, es posible mostrar que no son artefactos neutrales en tanto dan sentido a la acción estatal e inciden en la configuración de estrategias e instrumentos de intervención en el plano de la política pública. Por lo tanto, como artefactos simbólicos, las ideas ocupan un papel activo en reproducir la aceptación no solo de cierta racionalidad de la acción sino también de la posición y gobierno que alcanzan ciertos actores en las estructuras de decisión. Al mismo tiempo, como fue planteado inicialmente, las dificultades institucionales no necesariamente serían anomalías o fallas, sino más bien la expresión de la efectividad de los mecanismos de dominación y dependencia generados por una orientación y dirección política específica para alcanzar el desarrollo. Las experiencias neodesarrollistas podrían entenderse como una escena donde el caparazón estatal sufrió cambios que le otorgaron otro semblante y presencia frente a la sociedad, pero las ideas y axiomas que fundamentaron su accionar, al menos en el ámbito de política de desarrollo, se mantuvieron incólumes.

\section{Referencias}

Aguiar, D., Aristimuño, F. y Magrini, N. (2015). El rol del Banco Interamericano de Desarrollo (BID) en la reconfiguración de las instituciones y políticas de fomento a la ciencia, la tecnología y la innovación de la Argentina (1993-1999). Revista iberoamericana de ciencia tecnología y sociedad, 10(29), 11-40.

Aguirre, L. J. y Lo-Vuolo, R. (2013). Variedades de Capitalismo. Una aproximación al estudio comparado del capitalismo y sus aplicaciones para América Latina (documento de trabajo $n^{\circ}{ }^{85}$ ). Centro de Estudios Interdisciplinarios para el Estudio de Políticas Públicas

Albert, M. (1993). Capitalism against capitalism. John Wiley y Sons.

Aristimuño, F. y Lugones, M. (2019). El BID y las políticas de Ciencia, Tecnología e Innovación en Argentina (1990-2015). Ciencia, Tecnología y Política, 2(3), 1-12. https://doi.org/10.24215/26183188e031

Arrighi, G. (1978). Towards a theory of capitalist crisis. New Left Review, 11 1(3), 3-24.

Bair, J. (2005). Global Capitalism and Commodity Chains: Looking Back, Going Forward. Competition \& Change, 9(2), 153-180. https://doi.org/10.1179/102452905×45382

Bair, J. (2019). Class formation and commodity chains in the making of regional monocultures: Agrarian reform from above and below in Mexico's henequen and cotton zones. Journal of Agrarian Change, 19(3), 487-505. https://doi.org/10.1111/joac.12305

Baldwin, R. (2011). Trade and Industrialisation After Globalisation's 2nd Unbundling: How Building and Joining a Supply Chain Are Different and Why it Matters (Working Paper $n^{\circ}$ 17716). NBER. https://doi. org/10.3386/w17716

Baldwin, R. (2013). Global Supply Chains: Why They Emerged, Why They Matter, and Where They Are Going. En D. Elms y P. Low (Eds.), Global value chains in a changing world (pp. 13-59). World Trade Organization.

Baruj, G. y Porta, F. (2006). Políticas de competitividad en la Argentina y su impacto sobre la profundización del Mercosur. CEPAL.

Bekerman, M., Dulcich, F. y Gaite, P. (2018). La inserción comercial externa de la Argentina en la última década: su impacto sobre la estructura productiva. H-industri@: Revista de historia de la industria, los servicios y las empresas en América Latina, (23), 115-142.

Bekerman, M., Wiñazky, M. y Moncaut, N. (2013). Políticas productivas para fortalecer la inserción internacional de las empresas PYMES: Los consorcios de exportación en Argentina. Revista Integración y Comercio, $37(17), 57-73$. 
Belloni, P. y Wainer, A. (2013). La continuidad de la dependencia bajo nuevas formas: la relación entre la restricción externa y el capital extranjero en la Argentina. Cuadernos del Cendes, 30(83), 23-51.

BID -Banco Interamericano de Desarrollo-. (2005). El Grupo BID y la pequeña y mediana empresa (19902004). Resultados de 15 años de trabajo. Banco Interamericano de Desarrollo.

Blyde, J. (2014). Fábricas Sincronizadas. Banco Interamericano de Desarrollo.

Blyth, M. (2012). A Theory of Institutional Change. En M. Blyth (Ed.), Great Transformations. Economic Ideas and Institutional Change in the Twentieth Century (pp. 17-46). Cambridge University Press. https://doi. org/10.1017/cbo9781139087230.003

Boschi, R. (2011). Introdução: instituições, trajetórias e desenvolvimento-uma discussão a partir da América Latina. En R. Boschi (Ed.), Variedades de capitalismo, política e desenvolvimento na América Latina (pp. 7-30). Editora UFMG.

Boschi, R. y Gaitán, F. A. (2008). Empresas, capacidades estales y estrategias de desarrollo en Brasil, Argentina y Chile. Ponto de vista, (3), 1-51.

Bresser-Pereira, L. C. (2012). From Old to New Developmentalism in Latin America. En J. A. Ocampo y J. Ros (Eds.), The Oxford Handbook of Latin American Economics (pp. 108-129). Oxford Univertity Press. https://doi.org/10.1093/oxfordhb/9780199571048.013.0005

Bresser-Pereira, L. C. (2017). La nueva teoría desarrollista: una síntesis. Economía UNAM, 14(40), 48-66. https://doi.org/10.1016/j.eunam.2017.01.002

Chang, H. J. (2010). How to 'do' a developmental state: political, organisational and human resource requirements for the developmental state. En O. Edigheji (Ed.), Constructing a democratic developmental state in South Africa: potentials and challenges (pp. 82-96). HSRC Press.

Chibber, V. (2002). Bureaucratic Rationality and the Developmetal State. American Journal of Sociology, 107(4), 951-988. https://doi.org/10.1086/341010

Chibber, V. (2003). Locked in place: State-building and late industrialization in India. Princeton University Press.

Chodor, T. (2015). Neoliberal hegemony and the Pink Tide in Latin America. Breaking up with TINA? Palgrave. https://doi.org/10.1057/9781137444684

Cimoli, M., Castillo, M., Porcile, G. y Stumpo, G. (Eds.). (2017). Políticas industriales y tecnológicas en América Latina. CEPAL.

Cox, R. (2013). Fuerzas sociales, estados y órdenes mundiales: Más allá de la Teoría de Relaciones Internacionales. Relaciones internacionales, (24), 99-116.

Crespo, E. y Muñiz, M. (2017). Una aproximación a las condiciones globales del Desarrollo Económico. Revista Estado y Políticas Públicas, (8), 21-39.

Crotty, J. y Lee, K. K. (2005). From East Asian «miracle» to neo-liberal «mediocrity»: the effects of liberalization and financial opening on the post-crisis Korean economy. Global Economic Review, 34(4), 415-434. https://doi.org/10.1080/12265080500441388

Cypher, J. M. (2007). Shifting developmental paradigms in Latin America: Is neoliberalism history? En E. Pérez-Caldente y M. Vernengo (Eds.), Ideas, Policies and Economic Development in the Americas (pp. 31 61). Routledge. https://doi.org/10.4324/9780203964026

De-Mattos, C. (1979). Planes versus planificación en la experiencia latinoamericana. Revista de la CEPAL, (8), 79-96.

Dicken, P., Kelly, P. F., Olds, K. y Wai-Chung-Yeung, H. (2001). Chains and networks, territories and scales: towards a relational framework for analysing the global economy. Global Networks, 1(2), 89-112. https:// doi.org/10.1111/1471-0374.00007 
Sociedad y Economía N 42 (Ene - abr 2021) / e-ISSN: 2389-9050

Estado, estrategias de desarrollo y las ideas "correctas" en América Latina

Dini, M., Rovira, S. y Stumpo, G. (2014). Una promesa y un suspirar: políticas de innovación para PYMES en América Latina. CEPAL.

Diniz, E., Boschi, R. y Gaitán, F. (2012). Élites estratégicas y cambio institucional: la construcción del proyecto post-neoliberal en Argentina y Brasil. Revista de Estudos e Pesquisas sobre as Américas, 6(2), 14-53. https://doi.org/10.21057/repam.v6i2.8187

Ebenau, M. (2012). Varieties of Capitalism or Dependency? A Critique of the VoC Approach for Latin America. Competition \& Change, 16(3), 206-223. https://doi.org/10.1179/1024529412z.00000000014

Ebenau, M., Bruff, I. y May, C. (Eds.). (2015). New Directions in Comparative Capitalisms Research: Critical and Global Perspectives. Palgrave Macmillan. https://doi.org/10.1057/9781137444615

Féliz, M. (2015). Renta extraordinaria e industrialización en el neodesarrollismo. Límites y alternativas. Argentina, 2003-2012. Revista Economia Ensaios, 29(1), 7-24. https://doi.org/10.14393/reev29n1a2014-1

Fernández, V. R. (2001). Estrategias de desarrollo y transformación estatal. Buscando al Estado bajo el capitalismo global. Ediciones UNL.

Fernández, V. R. (2010). Desarrollo regional bajo transformaciones trans-escalares ¿Por qué y cómo recuperar la escala nacional? En V. R. Fernández y C. Brandão (Eds.), Escalas y políticas del desarrollo regional. Desafíos para América Latina (pp. 301-341). Editorial Miño y Dávila.

Fernández, V. R. (2015). Global Value Chains in Global Political Networks: Tool for Development or Neoliberal Device? Review of Radical Political Economics, 47(2), 209-230. https://doi. org/10.1177/0486613414532769

Fernández, V. R. (2017). La trilogía del erizo-zorro. Redes globales, trayectorias nacionales y dinámicas regionales desde la Periferia. Anthropos; Universidad Nacional del Litoral.

Fernández, V. R. y Brondino, G. (2018). Why does structuralism return to the forefront? En V. R. Fernández y G. Brondino (Eds.), Development in Latin America: Critical Discussions from the Periphery (pp. 11-45). Palgrave Macmillan. https://doi.org/10.1007/978-3-319-92183-9_2

Fernández, V. R. y Ebenau, M. (2018). Introducción. En V. R. Fernández y M. Ebenau (Eds.), Variedades de capitalismo entre centro y periferia. Miradas críticas desde América Latina (pp. 11-28). Miño y Dávila.

Fernández, V. R. y Trevignani, M. F. (2015). Cadenas Globales de Valor y Desarrollo: Perspectivas Críticas desde el Sur Global. Dados, 58(2), 499-536. https://doi.org/10.1590/00115258201551

Fernández, V. R. y Vigil, J. I. (2007). Clusters y desarrollo territorial. Revisión teórica y desafíos metodológicos para América Latina. Economía, Sociedad y Territorio, 6(24), 859-912. https://doi.org/10.22136/est002007241

Fernández, V. R., Amin, A. y Vigil, J. I. (2008). Discutiendo el desarrollo regional: desde la emergencia y la institucionalización de la nueva ortodoxia hacia su reconsideración. En V. R. Fernández, A. Amin y J. I. Vigil (Eds.), Repensando el desarrollo regional: contribuciones globales para una estrategia latinoamericana (pp. 19-61). Miño y Dávila.

Fernández, V. R., Bazza, A. y Seiler, C. (2017). Variedades Y Variaciones De Capitalismo En La Periferia. América Latina Y El Este Asiático Reconsiderados. Semestre Económico, 20(45), 77-108. https://doi. org/10.22395/seec.v20n45a3

Fernández, V. R., Ebenau, M. y Bazza, A. (2018). Rethinking Varieties of Capitalism from the Latin American Periphery. Review of Radical Political Economics, 50(2), 392-408. https://doi. org/10.1177/0486613417690139

Fernández, V. R., Güemes, M. C. y Vigil, J. I. (2006). Estado y desarrollo en los discursos del Banco Mundial. Problemas del desarrollo, 37(144), 33-71. 
Ferrando, A. (2013). Las Cadenas Globales de Valor, los Países en Desarrollo y sus PYMES. Instituto de Estrategia Internacional-CERA.

Furtado, C. (1974). Teoría y política del desarrollo económico. Siglo Veintiuno Editores.

Gaitán, F. y Boschi, R. (2016). Estado, Atores Predominantes e Coalizões para o Desenvolvimento: Brasil e Argentina em perspectiva comparada. En A. de-Ávila y R. Boschi (Eds.), Capacidades estatais em países emergentes: Brasil em perspectiva comparada (pp. 473-506). IPEA.

Gereffi, G. (2014). Global value chains in a post-Washington Consensus world. Review of International Political Economy, 21(1), 9-37. https://doi.org/10.1080/09692290.2012.756414

Gill, S. (1995). Globalisation, Market Civilisation, and Disciplinary Neoliberalism. Millennium - Journal of International Studies, 24(3), 399-423. https://doi.org/10.1177/03058298950240030801

Gill, S. (2012). Towards a radical concept of praxis: Imperial «common sense» versus the postmodern prince. Millennium: Journal of International Studies, 40(3), 505-524. https://doi. org/10.1177/0305829812442465

Gill, S. R. y Law, D. (1989). Global Hegemony and the Structural Power of Capital. International Studies Quarterly, 33(4), 475-499. https://doi.org/10.2307/2600523

Giuliani, E., Pietrobelli, C. y Rabellotti, R. (2005). Upgrading in global value chains: Lessons from Latin American clusters. World Development, 33(4), 549-573. https://doi.org/10.1016/j.worlddev.2005.01.002

Gore, C. (1996). Methodological nationalism and the misunderstanding of east asian industrialisation. European Journal of Development Research, 8(1), 77-122. https://doi.org/10.1080/09578819608426654

Hall, P. A. (1993). Policy Paradigms, Social Learning, and the State: The Case of Economic Policymaking in Britain. Comparative Politics, 25(3), 275-296. https://doi.org/10.2307/422246

Hall, P. y Soskice, D. (2001). An Introduction to Varieties of Capitalism. En P. Hall y D. Soskice (Eds.), Varieties of Capitalism. The Institutional Foundations of Comparative Advantage (pp. 1-70). Oxford University Press. https://doi.org/10.1093/0199247757.003.0001

Hernández, R., Martínez-Piva, J. M. y Mulder, N. (2014). Global value chains and world trade. Prospects and challenges for Latin America. CEPAL.

Hirschman, A. (1968). La economía política de la industrialización a través de la sustitución de importaciones en América Latina. El Trimestre Económico, 35(140-4), 625-658.

Jessop, B. (2016). The Developmental State in an Era of Finance-dominated Accumulation. En Y. Chu (Ed.), The Asian Developmental State (pp. 27-55). Palgrave Macmillan.

Kaplinsky, R. (2000). Globalisation and unequalisation: What can be learned from value chain analysis? Journal of Development Studies, 37(2), 117-146. https://doi.org/10.1080/713600071

Katz, C. (2015). ¿Qué es el neodesarrollismo? Una visión crítica. Argentina y Brasil. Revista Serviço Social y Sociedade, (122), 224-249. https://doi.org/10.1590/0101-6628.021

Lavarello, P. (2017). El (incompleto y breve) regreso de la política industrial: el caso de Argentina 20032015. Problemas del Desarrollo. Revista Latinoamericana de Economía, 48(190), 109-135. https://doi. org/10.22201/iiec.20078951e.2017.190.58275

Milberg, W. (2008). Shifting sources and uses of profits: Sustaining US financialization with global value chains. Economy and Society, 37(3), 420-451. https://doi.org/10.1080/03085140802172706

Monedero, J. C. (2019). Selectividad estratégica del Estado y el cambio de ciclo en América Latina. En H. Ouviña y M. Thwaites-Rey (Eds.), Estados en disputa. Auge y fractura del ciclo de impugnación al neoliberalismo en América Latina (pp. 338-376). CLACSO; El Colectivo. 
Sociedad y Economía N 42 (Ene - abr 2021) / e-ISSN: 2389-9050

Estado, estrategias de desarrollo y las ideas "correctas" en América Latina

Neilson, J. (2014). Value chains, neoliberalism and development practice: The Indonesian experience. Review of International Political Economy, 21 (1), 38-69. https://doi.org/10.1080/09692290.2013.809782

Peck, J. (2010). Economías políticas de escalas: políticas rápidas, relaciones interescalares y workfare neoliberal. En V. R. Fernández y C. Brandao (Eds.), Escalas y Políticas de desarrollo regional. Desafíos para América Latina (pp. 77-120). Miño y Dávila.

Pietrobelli, C. y Rabellotti, R. (2011). Global Value Chains Meet Innovation Systems: Are There Learning Opportunities for Developing Countries? World Development, 39(7), 1261-1269. https://doi. org/10.1016/j.worlddev.2010.05.013

Prebisch, R. (1986). El desarrollo económico de la América Latina y algunos de sus principales problemas. Desarrollo Económico, 26(103), 479-502. https://doi.org/10.2307/3466824

Schneider, B. R. (2009a). A comparative political economy of diversified business groups, or how states organize big business. Review of International Political Economy, 16(2), 178-201. https://doi. org/10.1080/09692290802453713

Schneider, B. R. (2009b). Hierarchical market economies and varieties of capitalism in Latin America. Journal of Latin American Studies, 41(3), 553-575. https://doi.org/10.1017/S0022216X09990186

Schneider, B. R. y Karcher, S. (2012). La política de las empresas en Latinoamérica: investigando sus estructuras, preferencias e influencia. Apuntes: Revista de Ciencias Sociales, 39(70), 7-28. https://doi. org/10.21678/apuntes.70.644

Seiler, C. (2020). La paradoja de la política industrial en Argentina. Jerarquización y ahuecamiento del Ministerio de Industria de la Nación (2009-2015). Colombia Internacional, (104), 95-129. https://doi. org/10.7440/colombiaint 104.2020 .04

Seiler, C. y Fernández, V. R. (2017). Política pyme y estructuras estatales en Argentina (2003-2015): Vínculos opacos y sombras inexploradas. Revista del CLAD Reforma y Democracia, (68), 205-240.

SELA -Sistema Económico Latinoamericano y del Caribe-. (2012). Cadenas de Valor, PYMES y Políticas Públicas. Experiencias Internacionales y Lecciones para América Latina y el Caribe. Sistema Económico Latinoamericano y del Caribe.

Sikkink, K. y Wolfson, L. (1993). Las capacidades y la autonomía del Estado en Brasil y la Argentina. Un enfoque neoinstitucionalista. Desarrollo Económico, 32(128), 544-577. https://doi.org/10.2307/3467177

Stezano, F. (2013). Políticas para la inserción de las microempresas y las pequeñas y medianas empresas en cadenas globales de valor en América Latina. CEPAL.

Sum, N. L. y Jessop, B. (2013). Towards a cultural political economy. Putting culture in its place in political economy. Edward Elgar.

Swyngedouw, E. (1997). Neither global nor local: «glocalization» and the politics of scale. Spaces of Globalization. En K. Cox (Ed.), Reasserting the Power of the Local (pp. 137-166). Guilford; Longman.

UNCTAD -United Nations Conference on Trade and Development-. (2010). Integrating Developing Countries' SMEs into Global Value Chains. United Nations.

Wade, R. (2006). Asfixia al Sur. New Left Review (español), (38), 101-114.

Weiss, L. (1998). The Myth of the Powerless State. Cornell University Press.

Werner, M., Bair, J. y Fernández, V. R. (2014). Linking Up to Development? Global Value Chains and the Making of a Post-Washington Consensus. Development and Change, 45(6), 1219-1247. https://doi.org/10.1111/ dech. 12132

Yeung, H. W. (2017). Rethinking the East Asian developmental state in its historical context: finance, geopolitics and bureaucracy. Area Development and Policy, 2(1), 1-23. https://doi.org/10.1080/237929 49.2016.1264868 